\title{
MACKEY FUNCTORS AND BISETS
}

\author{
I. HAMBLETON, L. R. TAYLOR, AND E. B. WILLIAMS
}

\begin{abstract}
For any finite group $G$, we define a bivariant functor from the Dress category of finite $G$-sets to the conjugation biset category, whose objects are subgroups of $G$, and whose morphisms are generated by certain bifree bisets. Any additive functor from the conjugation biset category to abelian groups yields a Mackey functor by composition. We characterize the Mackey functors which arise in this way.
\end{abstract}

\section{INTRODUCTION}

Let $G$ be a finite group. A Mackey functor in the sense of Dress [8, p. 301] is a bivariant functor

$$
\mathcal{M}: \mathcal{D}(G) \rightarrow \mathcal{A} b
$$

from the category of finite left $G$-sets and $G$-maps to abelian groups, satisfying a pull-back axiom (M1) and an additivity axiom (M2). These axioms express the classical Mackey properties from representation theory, as formulated by Green [9]. In this paper, unless otherwise mentioned, by a Mackey functor we mean a Mackey functor in the sense of Dress.

Many of the Mackey functors encountered in applications of the theory factor through the $G$-Burnside category $\mathbf{A}(G)$, whose objects are the subgroups $H \subset G$ and whose morphisms are generated by $H_{2}-H_{1}$ bisets with certain properties (compare [10, 1.A.4]). Let A. $(G)$ denote the additive completion of this category, defined in Section 3 ,

In Section $[6$ we define a subcategory B. $(G) \subset \mathbf{A} .(G)$, where the morphisms are restricted to be conjugation bisets, and construct a bivariant functor $j: \mathcal{D}(G) \rightarrow \mathbf{B}_{\bullet}(G)$ (see Definition 7.2). A Mackey functor $\mathcal{M}$ is said to be conjugation invariant provided that the centralizer $C_{G}(H)$ acts trivially on $M(G / H)$ for all $H \subset G$, via the $G$-maps $\varphi: G / H \rightarrow G / H$ given by $e H \mapsto z H$, for some $z \in C_{G}(H)$ (see Definition 3.1).

The main result (see Theorem 8.1) is a recognition principle for such Mackey functors.

Theorem A. Let $G$ be a finite group. A Mackey functor $\mathcal{M}: \mathcal{D}(G) \rightarrow \mathcal{A}$ b factors through $j: \mathcal{D}(G) \rightarrow \mathbf{B} .(G)$ if and only if $\mathcal{M}$ is conjugation invariant.

The applications surveyed in [10] and [12, mostly depend on the following immediate consequence of our main result.

Corollary B. For any additive functor $F:$ A. $(G) \rightarrow \mathcal{A} b$, the composition $F \circ j: \mathcal{D}(G) \rightarrow$ $\mathcal{A} b$ is a Mackey functor.

Date: Jan. 7, 2010 (revision).

Research partially supported by NSERC Discovery Grant A4000 and the NSF. The authors would also like to thank the SFB 478, Münster, for its hospitality and support in June 2008. 
This result applies to classical Mackey functors such as the Swan ring and the Dress ring, and to algebraic $K$-theory and $L$-theory functors encountered in geometric topology and surgery theory.

Remark 1.1. The topic of Mackey functors has been extensively explored over the last 30 years, and there is a large literature (see, for example, the survey by Webb [19]). Here are some remarks about a selection of this earlier work.

(a). The idea of reformulating the classical Mackey bivariant functor properties as a single functor out of an intermediate category has been carried out by several authors (see Lindner [15, Theorem 2], Gaunce Lewis [13], tom Dieck [7, Chap. IV.8], ThévenazWebb [17]).

(b). The use of bisets as morphisms in a category has also appeared in various settings in the literature (see, for example, Lewis-May-McClure [14], Adams-Gunawardena-Miller [1, §9, p. 454], Hambleton-Taylor-Williams [10, 1.A.4], and Bouc [2], [3, §2], [4]).

(c). In [8, pp. 292, 302] Dress restricts attention to a variant of classical Mackey functors, now usually called global Mackey functors [18, p. 267], defined on the category gr of finite groups and monomorphisms, and in particular the maps induced by conjugations depend only on the underlying group homomorphisms. In [8, p. 298-299], Dress describes the passage from global Mackey functors to his functors. The output of this process is a conjugation-invariant Mackey functor.

(d). In [18, p. 271], Webb sketches a proof of a result analogous to Theorem A, that a global Mackey functor is equivalent to an additive functor out of a category $\Omega_{\mathbf{Z}}$ whose objects are finite groups, and whose morphisms are bifree bisets. The restriction of $\Omega_{\mathbf{Z}}$ to the subgroups of a fixed group $G$ is just the category $\mathbf{A}_{\bullet}(G)$, defined in our MSRI preprint (1990) [11, Ex. 5.5].

(e). There is a sub-category of gr defined by restricting to the subgroups of a fixed group $G$, and to the monomorphisms generated by inclusions and conjugations. A Mackey functor out of this sub-category is equivalent to an additive functor out of $\mathbf{B} .(G)$. This is exactly the statement of Theorem A expressed in classical terms. In other words, Theorem A provides a direct method to check the Mackey properties, starting from minimal input data.

(f). The proof of Theorem A follows Webb's proof [18, p. 271] in outline, but we supply all the technical details necessary, for example, to check that we have actually constructed a functor $F:$ B. $(G) \rightarrow \mathcal{A} b$, factoring a given conjugation invariant Mackey functor.

\section{MACKEY FUNCTORS}

We will first recall the basic definitions Dress used in his formulation of induction theory [8]. Let $G$ be a finite group, and let $\mathcal{D}(G)$ denote the category whose objects are finite, left $G$-sets and whose morphisms are $G$-maps. A Mackey functor is a bivariant functor $\mathcal{M}=\left(\mathcal{M}_{*}, \mathcal{M}^{*}\right): \mathcal{D}(G) \rightarrow \mathcal{A} b$, where $\mathcal{A} b$ denotes the category of abelian groups and groups homomorphisms, such that $\mathcal{M}_{*}(S)=\mathcal{M}^{*}(S)$ for each object $S \in \mathcal{D}(G)$, and the following two properties hold: 
(M1) For any pullback diagram of finite $G$-sets

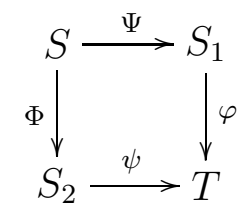

the induced maps give an commutative diagram

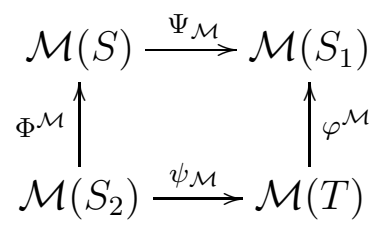

Here we denote the covariant maps by $\psi_{\mathcal{M}}$ and the contravariant maps by $\varphi^{\mathcal{M}}$.

(M2) The embeddings of $S_{1}$ and $S_{2}$ into the disjoint union $S_{1} \bigsqcup S_{2}$ define an isomorphism $\mathcal{M}^{*}\left(S_{1} \bigsqcup S_{2}\right) \rightarrow \mathcal{M}^{*}\left(S_{1}\right) \oplus \mathcal{M}^{*}\left(S_{2}\right)$. Let $\mathcal{M}(\emptyset)=0$.

The property (M1) is the usual double coset formula, and (M2) gives additivity. We remark that for any bivariant functor satisfying (M1), the composition $\mathcal{M}_{*}\left(S_{1}\right) \oplus \mathcal{M}_{*}\left(S_{2}\right) \rightarrow$ $\mathcal{M}_{*}\left(S_{1} \bigsqcup S_{2}\right)=\mathcal{M}^{*}\left(S_{1} \bigsqcup S_{2}\right) \rightarrow \mathcal{M}^{*}\left(S_{1}\right) \oplus \mathcal{M}^{*}\left(M_{2}\right)=\mathcal{M}_{*}\left(S_{1}\right) \oplus \mathcal{M}_{*}\left(S_{2}\right)$ is just the identity matrix. It follows that any sub-bivariant functor of a Mackey functor is Mackey.

Remark 2.1. We could replace the target category $\mathcal{A} b$ of abelian groups throughout by the category $R$-Mod of $R$-modules, for any commutative ring $R$ with unit.

\section{The $G$-Burnside CATEgory $\mathbf{A}(G)$}

We will be interested in Mackey functors which factor through the $G$-Burnside category $\mathbf{A}(G)$, whose objects are subgroups $H \subset G$, and where $\operatorname{Hom}_{\mathbf{A}(G)}\left(H_{1}, H_{2}\right)$ is the Grothendieck construction applied to the isomorphism classes of finite bifree $H_{2}-H_{1}$ bisets (meaning both left and right actions are free). In contrast, the morphisms in the $G$ Burnside category of [1, $\S 9$, p. 454], and our category $R G$-Morita [10, 1.A.4] just have a one-sided isotropy assumption.

To make our recognition principle more precise, we will define a subcategory $\mathbf{B}(G) \subset$ $\mathbf{A}(G)$ by restricting its morphisms to conjugation bisets (see Definition 6.1).

Because of the Grothendieck construction, $\mathbf{A}(G)$ and $\mathbf{B}(G)$ are both Ab-categories: the morphism sets are abelian groups and the compositions are bilinear [16, I.8, p. 28]. Let $u: \mathbf{A}(G) \rightarrow \mathbf{A} \bullet(G)$ and $u: \mathbf{B}(G) \rightarrow \mathbf{B} \bullet(G)$ denote the associated universal free additive categories, and the universal inclusions (see [16, VII.2, problem 6, p. 194]).

It turns out that the Mackey functors which factor through B. $(G)$ have an additional property, called conjugation invariance, which can be expressed in terms of their restriction to the orbit category $\operatorname{Or}(G)$. Recall that the objects of $\operatorname{Or}(G)$ are the subgroups $H \subset G$, and the morphisms $\operatorname{Hom}_{\mathbf{O r}(G)}\left(H_{1}, H_{2}\right)$ are the $G$-maps $\varphi: G / H_{1} \rightarrow G / H_{2}$. Any such $G$-map is uniquely determined by $e H_{1} \mapsto g H_{2}$, where $g^{-1} H_{1} g \subseteq H_{2}$. If $H_{1}=H_{2}=H$, then any element $z \in C_{G}(H)$ in the centralizer of $H$ gives a $G$-map $\varphi_{z}: G / H \rightarrow G / H$. 
Definition 3.1. A functor $F: \operatorname{Or}(G) \rightarrow \mathcal{A} b$ is called conjugation invariant if the induced maps $F\left(\varphi_{z}\right)=\mathrm{id}: F(H) \rightarrow F(H)$, whenever $\varphi_{z}: G / H \rightarrow G / H$ is given by $e H \mapsto z H$, for some $z \in C_{G}(H)$. A Mackey functor is said to be conjugation invariant if its restriction to $\operatorname{Or}(G)$ satisfies this condition.

We now relate this condition to the conjugation homomorphisms which will be used in the definition of $\mathbf{B} .(G)$. If $\varphi \in \operatorname{Hom}_{\mathbf{O r}(G)}\left(H_{1}, H_{2}\right)$ is represented by $g \in G$, we let $c_{g}: H_{1} \rightarrow$ $H_{2}$ denote the associated conjugation homomorphism given by $c_{g}\left(h_{1}\right)=g^{-1} h_{1} g \in H_{2}$, for all $h_{1} \in H_{1}$. Since the $G$-map $\varphi$ only depends on the coset $g H_{2}$, we may vary $g \sim g h_{2}$, for any $h_{2} \in H_{2}$. The associated conjugation homomorphism $c_{g h_{2}}=c_{h_{2}} \circ c_{g}$ is thus well-defined (as a homomorphism) up to conjugation by elements of $H_{2}$. Let

$$
c_{\varphi}:=\left[c_{g}\right] \in \operatorname{Hom}\left(H_{1}, H_{2}\right) /\left(\text { conjugation in } H_{2}\right)
$$

denote the equivalence class of the associated conjugation homomorphism to a $G$-map $\varphi\left(e H_{1}\right)=g H_{2}$. Two different morphisms $\varphi_{1}, \varphi_{2} \in \operatorname{Hom}_{\mathbf{O r}(G)}\left(H_{1}, H_{2}\right)$ yield the same equivalence class $c_{\varphi_{1}}=c_{\varphi_{2}}$ if and only if $g_{1} h_{2} g_{2}^{-1}$ centralizes $H_{1}$, for some $h_{2} \in H_{2}$.

Lemma 3.2. A functor $F: \operatorname{Or}(G) \rightarrow \mathcal{A} b$ is conjugation invariant if and only if $c_{\varphi_{1}}=c_{\varphi_{2}}$ implies $F\left(\varphi_{1}\right)=F\left(\varphi_{2}\right)$ for all morphisms $\varphi_{1}, \varphi_{2} \in \operatorname{Hom}_{\mathbf{O r}(G)}\left(H_{1}, H_{2}\right)$.

Proof. Suppose that $\varphi_{1}, \varphi_{2} \in \operatorname{Hom}_{\mathbf{O r}(G)}\left(H_{1}, H_{2}\right)$ are given by $\varphi_{i}\left(e H_{1}\right)=g_{i} H_{2}$, for $i=1,2$. If there exists an element $h_{2} \in H_{2}$ such that $z:=g_{1} h_{2} g_{2}^{-1} \in C_{G}\left(H_{1}\right)$, then we see that $\varphi_{1}=\varphi_{2} \circ \varphi_{z}$. Therefore, if $F$ is conjugation invariant and $c_{\varphi_{1}}=c_{\varphi_{2}}$ we conclude that $F\left(\varphi_{1}\right)=F\left(\varphi_{2}\right)$. Conversely, for any subgroup $H \subset G$, and any $z \in C_{G}(H)$, the $G$-maps $\varphi_{z}$, id: $G / H \rightarrow G / H$ have the property $c_{\varphi_{z}}=c_{\mathrm{id}}$. Therefore the given condition implies that $F\left(\varphi_{z}\right)=F(\mathrm{id})=\mathrm{id}$, and hence $F$ is conjugation invariant.

The morphisms in $\mathbf{A}(G)$ are defined by the Grothendieck construction with addition operation the disjoint union of bisets. By convention, the empty biset $\emptyset$ represents the zero element. Composition comes from the balanced product:

$$
{ }_{H_{3}} X_{H_{2}} \circ{ }_{H_{2}} Y_{H_{1}}=\left({ }_{H_{3}} X_{H_{2}}\right) \times{ }_{H_{2}}\left({ }_{H_{2}} Y_{H_{1}}\right) \text {. }
$$

The reader should check that this is well-defined on isomorphism classes of bisets and "bilinear" in that

$$
\left({ }_{H_{3}} X_{H_{2}} \bigsqcup{ }_{H_{3}} Y_{H_{2}}\right) \circ{ }_{H_{2}} Z_{H_{1}} \cong\left({ }_{H_{3}} X_{H_{2}} \circ{ }_{H_{2}} Z_{H_{1}}\right) \bigsqcup\left({ }_{H_{3}} Y_{H_{2}} \circ{ }_{H_{2}} Z_{H_{1}}\right)
$$

with a similar formula for disjoint union on the right. The morphisms in $\mathbf{A}$. $(G)$ are matrices of morphisms in $\mathbf{A}(G)$.

Definition 3.3. We define a contravariant involution $\tau: \mathbf{A}(G) \rightarrow \mathbf{A}(G)$, by the identity on objects, and on morphisms it is the map induced on the Grothendieck construction by the function which takes the finite bifree $H_{2}-H_{1}$ biset ${ }_{H_{2}} X_{H_{1}}$ to the finite bifree $H_{1}-H_{2}$ biset ${ }_{H_{1}} X_{H_{2}}$ which is $X$ as a set and $h_{1} \cdot x \cdot h_{2}$ is defined to be $h_{2}^{-1} x h_{1}^{-1}$.

The reader needs to check that isomorphic bisets are isomorphic after reversing the order, and should also check that the transpose conjugate of a disjoint union is isomorphic to the disjoint union of the conjugate transposes of the pieces. This means that $\tau$ is a functor which induces a homomorphism of Hom-sets. It is clearly an involution, not 
just up to natural equivalence. Since $\tau$ is a homomorphism on Hom-sets, it induces an additive contravariant involution $\tau^{\bullet}: \mathbf{A}_{\bullet}(G) \rightarrow \mathbf{A} \bullet(G)$, called conjugate transpose, which commutes with the functor $u: \mathbf{A}(G) \rightarrow \mathbf{A}_{\bullet}(G)$. By definition, $\tau^{\bullet}$ acts on a matrix of morphisms by applying $\tau$ to each entry, and then transposing the matrix.

\section{IndeCOMPOSABLE $H_{2}-H_{1}$ BISETS}

We will need some more detailed information about the bifree bisets used to define morphisms in $\mathbf{A}(G)$. Much of this material can be found in Bouc [2], but we include the details here to fix our notation and to emphasize the role of the base-points.

An $H_{2}-H_{1}$ biset is just a left $\left(H_{2} \times H_{1}^{\text {op }}\right)$-set and so any finite $H_{2}-H_{1}$-biset is a disjoint union of transitive $\left(H_{2} \times H_{1}^{\mathrm{op}}\right)$-sets. Since there are three groups acting $\left(H_{1}, H_{2}\right.$ and $H_{2} \times H_{1}^{\text {op }}$ ), the following definition should avoid confusion.

Definition 4.1. An $H_{2}-H_{1}$ biset $X$ is indecomposable if $X$ is a transitive $\left(H_{2} \times H_{1}^{\text {op }}\right)$-set.

Since every $H_{2}-H_{1}$ biset is a disjoint union of indecomposable $H_{2}-H_{1}$ bisets, it follows that $X_{1} \bigsqcup Y$ is isomorphic to $X_{1} \bigsqcup Y$ if and only if $X_{1}$ is isomorphic to $X_{2}$. One result of this remark is that the Grothendieck group of finite bifree $H_{2}-H_{1}$ bisets is the free abelian group on the indecomposable bifree ones.

An indecomposable $H_{2}-H_{1}$ biset $X$ is isomorphic (via choice of base point) to a coset space $\left(H_{2} \times H_{1}^{\mathrm{op}}\right) / S$, for some subgroup $S \subset H_{2} \times H_{1}^{\mathrm{op}}$. These models will be used extensively below, so we make some remarks and introduce some notation.

(i) To see $\left(H_{2} \times H_{1}^{\text {op }}\right) / S$ as an $H_{2}-H_{1}$ biset, define the $H_{2}$ action to be left multiplication in the first coordinate, but define $\left(h_{2}, h_{1}\right) S \cdot g_{1}=\left(h_{2}, g_{1}^{-1} h_{1}\right) S$.

(ii) We introduce the following notation for points in $\left(H_{2} \times H_{1}^{\mathrm{op}}\right) / S$ : if $h_{2} \in H_{2}$ and $h_{1} \in H_{1}$, write $\left\{h_{2}, h_{1}\right\}=\left(h_{2}, h_{1}^{-1}\right) S$. In this notation, the $H_{2}-H_{1}$ action is the evident one: $g_{2}\left\{h_{2}, h_{1}\right\} g_{1}=\left\{g_{2} h_{2}, h_{1} g_{1}\right\}$.

(iii) The left isotropy group of $(e, e)$ is just $\left(H_{2} \times\{e\}\right) \cap S$, and the right isotropy group of $(e, e)$ is just $\left(\{e\} \times H_{1}^{\text {op }}\right) \cap S$, so the actions are free if and only if $\left(H_{2} \times\{e\}\right) \cap S=\{e \times e\}$ and $\left(\{e\} \times H_{1}^{\text {op }}\right) \cap S=\{e \times e\}$.

(iv) Equivalently, the $S$-action is bifree if the compositions $\iota_{1}: S \subset H_{2} \times H_{1}^{\text {op }} \rightarrow H_{2}$ and $S \subset H_{2} \times H_{1}^{\text {op }} \rightarrow H_{1}^{\text {op }}$ are injective. We will work instead with the composite $\iota_{2}: S \rightarrow H_{2} \times H_{1}^{\text {op }} \rightarrow H_{1}^{\text {op }} \stackrel{\approx}{\rightarrow} H_{1}$, where the last homomorphism takes $h_{1}$ to $h_{1}^{-1}$.

(v) Let $L \subset \mathrm{H}_{2}$ denote the image of the isotropy subgroup $S$ under the injection $\iota_{1}$, and let $K \subset H_{1}$ denote the image of $S$ under the injection $\iota_{2}$. Define

$$
\gamma: L \stackrel{\iota_{1}^{-1}}{\longrightarrow} S \stackrel{\iota_{2}}{\longrightarrow} K
$$

and notice that this is an isomorphism.

(vi) Conversely, given subgroups $L \subset H_{2}, K \subset H_{1}$, and an isomorphism $\gamma: L \stackrel{\approx}{\rightarrow} K$, let ${ }_{H_{2}}[L, \gamma, K]_{H_{1}}$ denote the indecomposable bifree biset $\left(H_{2} \times H_{1}^{\mathrm{op}}\right) / S$, where $S \subset H_{2} \times H_{1}^{\text {op }}$ is the graph of

$$
\bar{\gamma}: L \stackrel{\gamma}{\rightarrow} K \subset H_{1} \stackrel{\iota}{\rightarrow} H_{1}^{\text {op }},
$$


and $\iota: H_{1} \stackrel{\approx}{\rightarrow} H_{1}^{\text {op }}$ is the usual isomorphism which takes $h$ to $h^{-1}$.

Given an indecomposable $H_{2}-H_{1}$ biset $X$, a choice of base-point $x \in X$ yields an isotropy subgroup $S_{x} \subset H_{2} \times H_{1}^{\text {op }}$ and a preferred biset isomorphism

$$
\Psi_{x}:\left(H_{2} \times H_{1}^{\mathrm{op}}\right) / S_{x} \cong X
$$

defined by $\Psi_{x}\left(\left\{h_{2}, h_{1}\right\}\right)=h_{2} x h_{1}$.

Definition 4.3 (The standard representation). Let $X$ be an indecomposable bifree $\mathrm{H}_{2}$ $H_{1}$ biset, and $x \in X$ a base-point. The standard representation for $X$ at $x$ is the data

$$
\Psi_{x}:_{H_{2}}\left[L_{x}, \gamma_{X, x}, K_{x}\right]_{H_{1}} \stackrel{\cong}{\rightarrow} X
$$

given by the preferred biset isomorphism $\Psi_{x}\left(\left\{h_{2}, h_{1}\right\}\right)=h_{2} x h_{1}$, where $L_{x} \subset H_{2}$ is the image of the isotropy subgroup $S_{x}$ under $\iota_{1}, K_{x} \subset H_{1}$ is the image of $\iota_{2}$, and

$$
\gamma_{X, x}: L_{x} \stackrel{\iota_{1}^{-1}}{\longrightarrow} S_{x} \stackrel{\iota_{2}}{\longrightarrow} K_{x}
$$

is an isomorphism. We noted above that $L_{x}, K_{x}$ and $\gamma_{X, x}$ determine $S_{x}$ as a (graph) subgroup of $H_{2} \times H_{1}^{\text {op }}$.

Remark 4.5. Any model coset space ${ }_{H_{2}}[L, \gamma, K]_{H_{1}}$ is the standard representation for some indecomposable bifree biset. We let $X=\left(H_{2} \times H_{1}^{\text {op }}\right) / S$, and choose $x \in X$ as the coset of the identity, then $L_{x}=L, K_{x}=K$ and $\gamma_{X, x}=\gamma$.

Remark 4.6. Here is a second description of the standard representation (compare [6, 5.1]). We present it so as to identify the component of any point $x$ in an $H_{2}-H_{1}$ bifree biset $X$, indecomposable or not. Let $L_{x}=\left\{h_{2} \in H_{2} \mid h_{2} x \in x H_{1}\right\}$. Check $L_{x}$ is a subgroup of $H_{2}$. Since the $H_{1}$ action is free, there exists a unique $h_{1} \in H_{1}$ such that $h_{2} x=x h_{1}$. Define a function $f: L_{x} \rightarrow H_{1}$ by $f\left(h_{2}\right)=h_{1}$. Let $K_{x}$ denote the image of $f$ and let $\gamma_{X, x}: L_{x} \rightarrow K_{x}$ denote the restriction of $f$. Check that $f$ is a homomorphism and hence $\gamma_{X, x}$ is an isomorphism. Finally check that $\Psi_{x}:_{H_{2}}\left[L_{x}, \gamma_{X, x}, K_{x}\right]_{H_{1}} \rightarrow X$ defined by Formula 4.4 above is an injection of $H_{2}-H_{1}$ bisets which is then automatically onto the component of $X$ containing $x$.

Hereafter we will call ${ }_{H_{2}}\left[L_{x}, \gamma_{X, x}, K_{x}\right]_{H_{1}}$ the standard representation of $X$ at $x$ even if $X$ is not indecomposable. It follows that

$$
\bigsqcup_{x \in H_{2} \backslash X / H_{1}} H_{2}\left[L_{x}, \gamma_{X, x}, K_{x}\right]_{H_{1}} \stackrel{\sqcup \Psi_{x}}{\longrightarrow} X
$$

is a bijection of $H_{2}-H_{1}$ bisets, where we take one $x$ in each indecomposable component of $X$. Here are two useful properties of the standard representation for the reader to verify.

We note the effect of the involution $\tau: \mathbf{A}(G) \rightarrow \mathbf{A}(G)$ on the standard representations.

Lemma 4.7. If $\left[L_{x}, \gamma_{X, x}, K_{x}\right]$ is the standard representation for $X$ at $x$, then $\left[K_{x}, \gamma_{X, x}^{-1}, L_{x}\right]$ is the standard representation for $\tau(X)$ at $x$. 
Next we consider a change of base-point. If $y$ is another point in the same indecomposable component as $x$, then $y=h_{2} x h_{1}$ for some choice of $h_{2} \in H_{2}$ and $h_{1} \in H_{1}$. For $g \in G$ and any subgroup $K \subset G$, let $K^{g}=g^{-1} K g$. If $\gamma: L \rightarrow K$ is a homomorphism between subgroups of $G$ and if $g_{1}, g_{2} \in G$, define ${ }_{g_{2}} \gamma^{g_{1}}(h)=g_{1}^{-1} \gamma\left(g_{2}^{-1} h g_{2}\right) g_{1}: L^{g_{2}^{-1}} \rightarrow K^{g_{1}}$.

Lemma 4.8. With notation as above, the standard representation for $X$ at $y=h_{2} x h_{1} \in X$ is $\left[L_{x}^{h_{2}^{-1}},{ }_{h_{2}} \gamma_{X, x}^{h_{1}}, K_{x}^{h_{1}}\right]$. In other words, $L_{y}=L_{x}^{h_{2}^{-1}}, K_{y}=K_{x}^{h_{1}}$ and $\gamma_{X, y}={ }_{h_{2}} \gamma_{X, x}^{h_{1}}$.

It will be useful later to be able to identify $X / H_{1}$ and $H_{2} \backslash X$.

Lemma 4.9. If $X$ is an indecomposable, bifree $H_{2}-H_{1}$ biset, then the bijection of bisets $\Psi_{x}:_{H_{2}}\left[L_{x}, \gamma_{X, x}, K_{x}\right]_{H_{1}} \rightarrow X$ induces

(i) a bijection of left $H_{2}$-sets $H_{2} / L_{x} \rightarrow X / H_{1}$

(ii) a bijection of right $H_{1}$-sets $K_{x} \backslash H_{1} \rightarrow H_{2} \backslash X$.

Proof. The proof is immediate.

\section{Composition of Bisets}

Fix indecomposable, bifree bisets ${ }_{H_{2}}\left(X_{1}\right)_{H_{1}}$ and ${ }_{H_{3}}\left(X_{2}\right)_{H_{2}}$ and let us analyze $X_{3}=$ $X_{2} \times_{H_{2}} X_{1}$. In general $X_{3}$ will not be indecomposable, and the Mackey double coset formula enters the picture. We begin by analyzing the standard representation at a point.

Lemma 5.1. The standard representation for $X_{3}=X_{2} \times_{H_{2}} X_{1}$ at $\left[x_{2}, x_{1}\right]$ is given by

- $L_{\left[x_{2}, x_{1}\right]}=\gamma_{X_{2}, x_{2}}^{-1}\left(K_{x_{2}} \cap L_{x_{1}}\right)$,

- $K_{\left[x_{2}, x_{1}\right]}=\gamma_{X_{3},\left[x_{2}, x_{1}\right]}\left(L_{\left[x_{2}, x_{1}\right]}\right)=\gamma_{X_{1}, x_{1}}\left(K_{x_{2}} \cap L_{x_{1}}\right)$

- $\gamma_{X_{3},\left[x_{2}, x_{1}\right]}=\gamma_{X_{1}, x_{1}} \circ \gamma_{X_{2}, x_{2}}$.

Proof. Since the projection $X_{2} \times X_{1} \rightarrow X_{2} \times_{H_{2}} X_{1}$ is onto, every point in $X_{2} \times_{H_{2}} X_{1}$ is the image of a point in $X_{2} \times X_{1}$. Given $x_{1} \in X_{1}$ and $x_{2} \in X_{2}$ write $\left[x_{2}, x_{1}\right] \in X_{3}=X_{2} \times_{H_{2}} X_{1}$ for the image of $x_{2} \times x_{1}$. In this notation, the $H_{3}-H_{1}$ structure is the evident one: $h_{3}\left[x_{2}, x_{1}\right] h_{1}=\left[h_{3} x_{2}, x_{1} h_{1}\right]$.

The point $\left[x_{2}, x_{1}\right] \in X_{3}$ is a set of points $\left\{x_{2} h \times h^{-1} x_{1}\right\} \subset X_{2} \times X_{1}$ for all $h \in H_{2}$. If $h_{3}\left(x_{2} \times x_{1}\right) h_{1}=x_{2} h \times h^{-1} x_{1} \in X_{2} \times X_{1}$ for some $h \in H_{2}$ then $h_{3} x_{2}=x_{2} h$ and $h^{-1} x_{1}=x_{1} h_{1}$. Now if $h_{3} x_{2}=x_{2} h$ then $h=\gamma_{x_{2}, x_{2}}\left(h_{3}\right)$ and $h_{3} \in L_{x_{2}}$. If $h^{-1} x_{1}=x_{1} h_{1}$ then $h_{1}=\gamma_{x_{1}, x_{1}}\left(h^{-1}\right)$ and $h^{-1} \in L_{x_{1}}$. Since $L_{x_{1}}$ is a group, $h \in L_{x_{1}}$. Since $h=\gamma_{x_{2}, x_{2}}\left(h_{3}\right)$, $h \in K_{x_{2}}$. Therefore, $h_{3} \in \gamma_{X_{2}, x_{2}}^{-1}\left(K_{x_{2}} \cap L_{x_{1}}\right)$. Let $L_{\left[x_{2}, x_{1}\right]}=\gamma_{X_{2}, x_{2}}^{-1}\left(K_{x_{2}} \cap L_{x_{1}}\right)$ and define $\gamma_{X_{3},\left[x_{2}, x_{1}\right]}=\gamma_{X_{1}, x_{1}} \circ \gamma_{X_{2}, x_{2}}$. What we have seen so far is that if $h_{3}\left[x_{2}, x_{1}\right] h_{1}=\left[x_{2}, x_{1}\right]$ in $X_{3}$, then $h_{3} \in L_{\left[x_{2}, x_{1}\right]}$ and $h_{1}=\gamma_{X_{3},\left[x_{2}, x_{1}\right]}\left(h_{3}\right)$.

If $h_{3} \in L_{\left[x_{2}, x_{1}\right]}$, then $h_{3}\left(x_{2} \times x_{1}\right) \gamma_{X_{3},\left[x_{2}, x_{1}\right]}\left(h_{3}\right)=x_{2} \gamma_{x_{2}, x_{2}}\left(h_{3}\right) \times \gamma_{X_{2}, x_{2}}\left(h_{3}\right)^{-1} x_{1}$ and it further follows that for any $h_{2} \in H_{2}, h_{3}\left(x_{2} h_{2} \times h_{2}^{-1} x_{1}\right) \gamma_{X_{3},\left[x_{2}, x_{1}\right]}\left(h_{3}\right)=x_{2} \gamma_{X_{2}, x_{2}}\left(h_{3}\right) h_{2} \times$ $h_{2}^{-1} \gamma_{X_{2}, x_{2}}\left(h_{3}\right)^{-1} x_{1}=x_{2}\left(\gamma_{X_{2}, x_{2}}\left(h_{3}\right) h_{2}\right) \times\left(\gamma_{X_{2}, x_{2}}\left(h_{3}\right) h_{2}\right)^{-1} x_{1}$. In other words, if $L_{\left[x_{2}, x_{1}\right]}$ and $\gamma_{X_{3},\left[x_{2}, x_{1}\right]}$ are defined as above, then for any $h_{3} \in L_{\left[x_{2}, x_{1}\right]}, h_{3}\left[x_{2}, x_{1}\right] \gamma_{X_{3},\left[x_{2}, x_{1}\right]}\left(h_{3}\right)=\left[x_{2}, x_{1}\right]$. If we let $K_{\left[x_{2}, x_{1}\right]}=\gamma_{X_{3},\left[x_{2}, x_{1}\right]}\left(L_{\left[x_{2}, x_{1}\right]}\right)$, then the proof is complete. 
Lemma 5.2. The double coset space $K_{x_{2}} \backslash H_{2} / L_{x_{1}}$ parametrizes the indecomposable components of $X_{2} \times_{H_{2}} X_{1}$, where $x_{1} \in X_{1}$ and $x_{2} \in X_{2}$ are base points.

Proof. To simplify the notation let $X_{2}={ }_{H_{2}}\left[L_{3}, \gamma_{2}, K_{2}\right]_{H_{1}}$, and $X_{1}={ }_{H_{2}}\left[L_{2}, \gamma_{1}, K_{1}\right]_{H_{1}}$. The set of indecomposable components is given by

$$
H_{3} \backslash\left(X_{2} \times_{H_{2}} X_{1}\right) / H_{1}=\left(H_{3} \backslash X_{2}\right) \times_{H_{2}}\left(X_{1} / H_{1}\right) .
$$

By Lemma 4.9, $\left(H_{3} \backslash X_{2}\right) \times_{H_{2}}\left(X_{1} / H_{1}\right)=\left(K_{2} \backslash H_{2}\right) \times_{H_{2}}\left(H_{2} / L_{2}\right)=K_{2} \backslash H_{2} / L_{2}$. If $\left\{h_{2}\right\} \in$ $K_{2} \backslash H_{2} / L_{2}$ is a set of double coset representatives with $h_{2} \in H_{2}$, then $\left[x_{2} h_{2}, x_{1}\right]$ gives a set of points, one in each indecomposable component.

Next we give an explicit formula for $X_{3}=X_{2} \times_{H_{2}} X_{1}$ as a disjoint union of indecomposable bisets (compare [2, 3.2]). We define a map

$$
\Phi_{h_{2}}:{ }_{H_{3}}\left[L_{x_{3}}, \gamma_{X_{3}, x_{3}}, K_{x_{3}}\right]_{H_{1}} \rightarrow_{H_{3}}\left[L_{x_{2}}, \gamma_{X_{2}, x_{2}}, K_{x_{2}}\right]_{H_{2}} \times_{H_{2} H_{2}}\left[L_{x_{1}}, \gamma_{X_{1}, x_{1}}, K_{x_{1}}\right]_{H_{1}}
$$

by the formula $\Phi_{h_{2}}\left(\left\{h_{3}, h_{1}\right\}\right)=\left[\left\{h_{3}, h_{2}\right\},\left\{e, h_{1}\right\}\right]$, using the notation of Definition 4.3.

Theorem 5.3 (Mackey double coset formula). The $H_{3}-H_{1}$ biset $X_{3}=X_{2} \times_{H_{2}} X_{1}$ is given by the disjoint union of the left-hand vertical maps in the diagram

$$
\begin{gathered}
{\left[\gamma_{X_{2}, x_{2}}^{-1}\left(K_{x_{2}} \cap L_{x_{1}}^{h_{2}^{-1}}\right), \gamma_{X_{1}, x_{1}} \circ c_{h_{2}} \circ \gamma_{X_{2}, x_{2}}, \gamma_{X_{1}, x_{1}}\left(K_{x_{2}}^{h_{2}} \cap L_{x_{1}}\right)\right]={ }_{H_{3}}\left[L_{x_{3}}, \gamma_{X_{3}, x_{3}}, K_{x_{3}}\right]_{H_{1}}} \\
\downarrow_{\Phi_{h_{2}}} \downarrow_{\Psi_{x_{3}}} \\
{ }_{H_{3}}\left[L_{x_{2}}, \gamma_{X_{2}, x_{2}}, K_{x_{2}}\right]_{H_{2}} \times_{H_{2} H_{2}}\left[L_{x_{1}}, \gamma_{X_{1}, x_{1}}, K_{x_{1}}\right]_{H_{1}} \frac{\Psi_{x_{2}} \times \Psi_{x_{1}}}{\approx} X_{2} \times_{H_{2}} X_{1}
\end{gathered}
$$

over the base-points $x_{3}=\left[x_{2} h_{2}, x_{1}\right]$, for $h_{2} \in K_{x_{2}} \backslash H_{2} / L_{x_{1}}$.

Proof. We first derive the explicit formula displayed in the top left corner of the diagram. Let $\left[L_{x_{2}}, \gamma_{X_{2}, x_{2}}, K_{x_{2}}\right]$ be the standard representation for $X_{2}$ at $x_{2}$, and let $\left[L_{x_{1}}, \gamma_{X_{1}, x_{1}}, K_{x_{1}}\right]$ be the standard representation for $X_{1}$ at $x_{1}$. Then the standard representation for $X_{2}$ at $x_{2} h_{2}$ is given by Lemma 4.8: $\left[L_{x_{2}}, \gamma_{X_{2}, x_{2} h_{2}}, K_{x_{2}}\right]=\left[L_{x_{2}}, \gamma_{X_{2}, x_{2}}^{h_{2}}, K_{x_{2}}^{h_{2}}\right]$. It further follows that the standard representation for $X_{3}=X_{2} \times_{H_{2}} X_{1}$ at $x_{3}=\left[x_{2} h_{2}, x_{1}\right]$ is given by

$$
\left[L_{x_{3}}, \gamma_{X_{3}, x_{3}}, K_{x_{3}}\right]=\left[L_{\left[x_{2} h_{2}, x_{1}\right]}, \gamma_{X_{3},\left[x_{2} h_{2}, x_{1}\right]}, K_{\left[x_{2} h_{2}, x_{1}\right]}\right] .
$$

By Lemmas 4.8, 5.1 and 5.2

$$
\begin{aligned}
& \text { - } L_{\left[x_{2} h_{2}, x_{1}\right]}=\gamma_{X_{2}, x_{2} h_{2}}^{-1}\left(K_{x_{2} h_{2}} \cap L_{x_{1}}\right)=\left(\gamma_{X_{2}, x_{2}}^{h_{2}}\right)^{-1}\left(K_{x_{2}}^{h_{2}} \cap L_{x_{1}}\right)=\gamma_{X_{2}, x_{2}}^{-1}\left(K_{x_{2}} \cap L_{x_{1}}^{h_{2}^{-1}}\right) \\
& \text { - } K_{\left[x_{2} h_{2}, x_{1}\right]}=\gamma_{X_{1}, x_{1}}\left(K_{x_{2} h_{2}} \cap L_{x_{1}}\right)=\gamma_{X_{1}, x_{1}}\left(K_{x_{2}}^{h_{2}} \cap L_{x_{1}}\right) \\
& \text { - } \gamma_{X_{3},\left[x_{2} h_{2}, x_{1}\right]}=\gamma_{X_{1}, x_{1}} \circ \gamma_{X_{2}, x_{2} h_{2}}=\gamma_{X_{1}, x_{1}} \circ \gamma_{X_{2}, x_{2}}^{h_{2}}=\gamma_{X_{1}, x_{1}} \circ c_{h_{2}} \circ \gamma_{X_{2}, x_{2}}
\end{aligned}
$$


For $h_{2} \in H_{2}$ the function defined by $\Phi_{h_{2}}\left(\left\{h_{3}, h_{1}\right\}\right)=\left[\left\{h_{3}, h_{2}\right\},\left\{e, h_{1}\right\}\right]$ makes the following diagram commute

$$
\begin{aligned}
& { }_{H_{3}}\left[L_{\left[x_{2} h_{2}, x_{1}\right]}, \gamma_{\left.X_{3}, x_{2} h_{2}, x_{1}\right]}, K_{\left[x_{2} h_{2}, x_{1}\right]}\right]_{H_{1}} \stackrel{\Phi_{h_{2}}}{\longrightarrow} H_{3}\left[L_{x_{2}}, \gamma_{X_{2}, x_{2}}, K_{x_{2}}\right]_{H_{2}} \times_{H_{2} H_{2}}\left[L_{x_{1}}, \gamma_{X_{1}, x_{1}}, K_{x_{1}}\right]_{H_{1}} \\
& \longrightarrow \Psi_{\left[x_{2} h_{2}, x_{1}\right]}{ }_{H_{3}}\left(X_{2} \times_{H_{2}} X_{1}\right)_{H_{1}}
\end{aligned}
$$

Since the $\Psi$ maps are injective, it suffices to prove that the formula given for $\Phi_{h_{2}}$ makes the diagram commute. Over and then down takes $\left\{h_{3}, h_{1}\right\}$ to $\left[\left\{h_{3}, h_{2}\right\},\left\{e, h_{1}\right\}\right]$ and then to $\left[h_{3} x_{2} h_{2}, x_{1} h_{1}\right]$ whereas $\Psi_{\left[x_{2} h_{2}, x_{1}\right]}\left(\left\{h_{3}, h_{1}\right\}\right)=h_{3}\left[x_{2} h_{2}, x_{1}\right] h_{1}=\left[h_{3} x_{2} h_{2}, x_{1} h_{1}\right]$.

The conclusion now follows immediately from the commutativity of the displayed diagram, since the disjoint union of the right-hand vertical maps $\Psi_{x_{3}}$ is a bijection, and the map $\Psi_{x_{2}} \times \Psi_{x_{1}}$ is a bijection (see Definition 4.3).

Remark 5.4. The map $\gamma_{X_{3},\left[x_{2} h_{2}, x_{1}\right]}$ can be displayed as the composition on the top row of the diagram

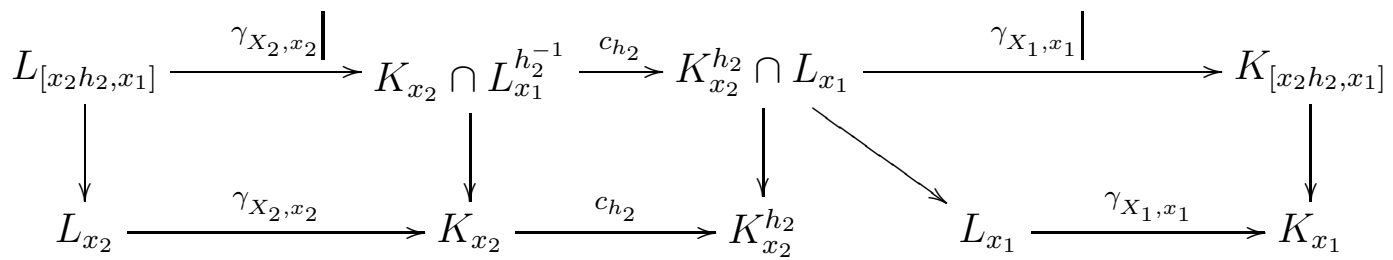

where $c_{g}(h)=g^{-1} h g$ is conjugation.

\section{Conjugation bisets and B $(G)$}

We will now define a subcategory $\mathbf{B}(G) \subset \mathbf{A}(G)$, with the same objects (the subgroups of $G$ ), but with morphisms restricted to bifree conjugation bisets. As before, we perform the Grothendieck construction on the isomorphism classes of these bisets to get an $\mathcal{A} b$-category. The universal construction $u: \mathbf{B}(G) \rightarrow \mathbf{B}_{\bullet}(G)$ then produces an additive subcategory (with involution) of $\mathbf{A} \cdot(G)$.

Definition 6.1 (Conjugation bisets). An indecomposable conjugation biset is an indecomposable bifree biset ${ }_{H_{2}} X_{H_{1}}$ so that the isomorphism in the standard representation of $X$ at $x \in X, \gamma_{X, x}=c_{g}$, for some $g \in G$ such that $g^{-1} L_{x} g=K_{x}$. A conjugation biset is a bifree biset, each of whose indecomposable subsets satisfies this condition.

Remark 6.2. The choice of $g$ such that $\gamma_{X, x}=c_{g}$ is not unique, but the conjugation biset only depends on $\gamma_{X, x}$ as a homomorphism. This is the basic reason that functors out of $\mathbf{B}(G)$ are conjugation invariant. By definition, $c_{g}\left(h_{2}\right)=g^{-1} h_{2} g \in K_{x}$, for all $h_{2} \in L_{x} \subset H_{2}$, where $g^{-1} L_{x} g=K_{x} \subset H_{1}$. We have $c_{g}=c_{g_{1}}: L_{x} \rightarrow K_{x}$ if and only if $g_{1} g^{-1} \in C_{G}\left(L_{x}\right)$, where $C_{G}\left(L_{x}\right)$ denotes the centralizer of $L_{x}$ in $G$.

The formula for the change of base-point in Lemma 4.8 shows that the definition of conjugation biset does not depend on the choice of point $x$ used to compute $\gamma_{X, x}$ : the element giving the conjugation may change, but not the fact that it is given by some 
conjugation. In particular, any biset which is isomorphic to a conjugation biset is itself a conjugation biset.

By Theorem 5.3, the composition of two conjugation bisets is again a conjugation biset. In addition, by Lemma 4.7, the involution $\tau$ restricts to give an involution $\tau: \mathbf{B}(G) \rightarrow$ $\mathbf{B}(G)$.

§6A. Induction and restriction. There are two extreme cases of indecomposable bifree bisets ${ }_{H_{2}} X_{H_{1}}$.

(i) We say ${ }_{H_{2}} X_{H_{1}}$ is a restriction if the $H_{1}$ action is transitive.

(ii) We say ${ }_{H_{2}} X_{H_{1}}$ is an induction if the $H_{2}$ action is transitive.

We say that ${ }_{H_{2}} X_{H_{1}}$ is an isomorphism if it is both a restriction and an induction.

Proposition 6.3. Let ${ }_{H_{2}} X_{H_{1}}$ be an indecomposable, bifree biset. Pick a point $x \in X$ and let $\left[L_{x}, \gamma_{X, x}, K_{x}\right]$ be the standard representation.

(i) ${ }_{H_{2}} X_{H_{1}}$ is a restriction if and only if $K_{x}=H_{1}$.

(ii) ${ }_{\mathrm{H}_{2}} \mathrm{X}_{\mathrm{H}_{1}}$ is an induction if and only if $L_{x}=H_{2}$.

Proof. Immediate from Lemma 4.9,

Proposition 6.4. If ${ }_{H_{2}} X_{H_{1}}$ is an isomorphism, $\tau\left({ }_{H_{2}} X_{H_{1}}\right)$ is the inverse isomorphism. Conversely, if ${ }_{\mathrm{H}_{2}} X_{\mathrm{H}_{1}}$ has an inverse then it is an isomorphism. This justifies the terminology.

Proof. Let ${ }_{H_{1}} Y_{H_{2}}$ be the inverse for ${ }_{H_{2}} X_{H_{1}}$. Then ${ }_{H_{2}} X_{H_{1}} \times{ }_{H_{1} H_{1}} Y_{H_{2}}$ is isomorphic to ${ }_{\mathrm{H}_{2}} \mathrm{H}_{2} \mathrm{H}_{2}$. Since $\left({ }_{\mathrm{H}_{2}} \mathrm{H}_{2} \mathrm{H}_{2}\right) / \mathrm{H}_{2}$ is a point, so is $\left({ }_{\mathrm{H}_{2}} \mathrm{X}_{\mathrm{H}_{1}} \times{ }_{\mathrm{H}_{1} \mathrm{H}_{1}} Y_{H_{2}}\right) / H_{2}=\left(X_{1} / H_{1}\right) \times\left(Y / H_{2}\right)$. It follows that $Y$ is a restriction. The other equation for the inverse shows that $Y$ is also an induction.

If ${ }_{H_{2}} X_{H_{1}}$ is both an induction and a restriction, it follows that the standard representation is $H_{2}, H_{1}$ and some isomorphism $\gamma_{X, x}$. By Lemma 4.7 the standard representation for $\tau(X)$ is $\left[H_{1}, \gamma_{X, x}^{-1}, H_{2}\right]$. By Theorem [5.3, $\tau(X)$ is the inverse for $X$.

It further follows from Theorem 5.3 that the composition of two restrictions is a restriction and the composition of two inductions is an induction. More explicitly, we have the following.

Proposition 6.5. Let $\left[L_{x_{1}}, \gamma_{X_{1}, x_{1}}, K_{x_{1}}\right]$ be the standard representation for the biset ${ }_{H_{2}} X_{1_{H_{1}}}$ at $x_{1}$ and let $\left[L_{x_{2}}, \gamma_{X_{2}, x_{2}}, K_{x_{2}}\right]$ be the standard representation for the biset ${ }_{H_{3}} X_{2_{H_{2}}}$ at $x_{2}$.

(i) If $X_{1}$ and $X_{2}$ are both restrictions then so is $X_{2} \times_{H_{2}} X_{1}$.

(ii) If $X_{1}$ and $X_{2}$ are both inductions then so is $X_{2} \times_{H_{2}} X_{1}$.

(iii) If $X_{1}$ is an induction and if $X_{2}$ is a restriction then $\left[L_{x_{2}}, \gamma_{X_{1}, x_{1}} \circ \gamma_{X_{2}, x_{2}}, K_{x_{1}}\right]$ is the standard representation for the composition using the point $\left[x_{2}, x_{1}\right]$

Proof. This follows from Lemma 5.1 and some standard set theory.

Definition 6.6. We define three subcategories of $\mathbf{A}(G)$, denoted $\operatorname{Res}_{\mathbf{A}}(G), \operatorname{Ind}_{\mathbf{A}}(G)$ and $\mathrm{Iso}_{\mathbf{A}}(G)$. The objects of any of these categories are all the objects of $\mathbf{A}(G)$. The morphisms in $\operatorname{Res}_{\mathbf{A}}(G)$ are the set of all restrictions, the morphisms in $\operatorname{Ind}_{\mathbf{A}}(G)$ are the set of all inductions and the morphisms in $\operatorname{Iso}_{\mathbf{A}}(G)$ are the isomorphisms. 
Remark 6.7. Because $\operatorname{Hom}_{\mathbf{A}(G)}\left(H_{1}, H_{2}\right)$ is the free abelian group on the indecomposable bifree $H_{2}-H_{1}$ bisets, $\operatorname{Hom}_{\operatorname{Res}(G)}\left(H_{1}, H_{2}\right) \subset \operatorname{Hom}_{\mathbf{A}(G)}\left(H_{1}, H_{2}\right)$, and indeed it is a summand. There is a similar statement for each of $\operatorname{Ind}(G)$ and $\operatorname{Iso}(G)$. Moreover, $\operatorname{Iso}(G)=\operatorname{Res}(G) \cap$ $\operatorname{Ind}(G)$.

Let ${ }_{H_{2}} X_{H_{1}}$ be an indecomposable bifree biset. After choosing a point $x \in X$, we can display $X$ as a composition.

Definition 6.8. Let $\left[L_{x}, \gamma_{X, x}, K_{x}\right]$ be the standard representation for $X$ at $x$. We may regard $H_{2}$ is an $H_{2}-L_{x}$ biset, and $H_{1}$ as a $K_{x}-H_{1}$ biset, via group multiplication in $G$.

(i) We define $\operatorname{Ind}_{x}={ }_{H_{2}}\left(H_{2}\right)_{L_{x}}$, note that it is an induction and call it the standard induction for $X$ at $x$.

(ii) We define $\operatorname{Res}_{x}={ }_{K_{x}}\left(H_{1}\right)_{H_{1}}$, note that it is a restriction and call it the standard restriction for $X$ at $x$.

(iii) There are two evident isomorphisms. Make $K_{x}$ into a left $L_{x}$ set using $\gamma_{X, x}$, and into a right $K_{x}$ set via group multiplication. This makes $K_{x}$ into an $L_{x}-K_{x}$ biset. Similarly make $L_{x}$ into an $L_{x}-K_{x}$ biset using $\gamma_{X, x}^{-1}$. Let ${ }_{L_{x}}\left(K_{x}\right)_{K_{x}}$ be denoted $\mathfrak{R}_{x}$ and let ${ }_{L_{x}}\left(L_{x}\right)_{K_{x}}$ be denoted $\mathfrak{L}_{x}$.

Note that $\mathfrak{L}_{x}$ and $\mathfrak{R}_{x}$ are isomorphic as bisets via the bijection $\gamma_{X, x}: L_{x} \rightarrow K_{x}$. The following composition formula was also observed in [2, Lemme 3] and [5, 7.4].

Proposition 6.9. As $\mathrm{H}_{2}-\mathrm{H}_{1}$ bisets, an indecomposable bifree biset $\mathrm{X}$ is isomorphic to the composition $\operatorname{Ind}_{x} \circ \mathfrak{L}_{x} \circ \operatorname{Res}_{x}$.

(i) The standard restriction $\operatorname{Res}_{x}$ is always a conjugation restriction.

(ii) The standard induction $\operatorname{Ind}_{x}$ is always a conjugation induction.

(iii) The indecomposable bifree biset $X$ is a conjugation biset if and only if $\mathfrak{L}_{x}$ is a conjugation isomorphism.

(iv) Moreover, $X$ is a restriction if and only if $\operatorname{Ind}_{x}$ is the identity; $X$ is an induction if and only if $\operatorname{Res}_{x}$ is the identity.

Proof. The standard representation for $\operatorname{Ind}_{x}$ is ${ }_{H_{2}}\left[L_{x}, i d, L_{x}\right]_{L_{x}}$, so it is clearly a conjugation biset. The case $\operatorname{Res}_{x}$ is similar. The standard representation for $\mathfrak{R}_{x}$ is ${ }_{L_{x}}\left[L_{x}, \gamma_{X, x}, K_{x}\right]_{K_{x}}$, and so $\mathfrak{R}_{x}$ is a conjugation biset if and only if $X$ is.

The function

$$
\operatorname{Ind}_{x} \times_{L_{x}} \mathfrak{L}_{x} \times_{K_{x}} \operatorname{Res}_{x} \rightarrow X
$$

which sends the image of $h_{2} \times \ell \times h_{1}$ to $h_{2} \ell x h_{1}$ can be checked to be a bijection of bisets. The remarks about $X$ being a restriction or an induction are immediate.

Remark 6.10. Of course the choice of $x \in X$ is not unique, so let $y=h_{2} x h_{1}$. Let $\operatorname{Res}_{y}$, Ind $y$ and $\mathfrak{R}_{y}$ be the corresponding bisets. Define an $L_{y}-L_{x}$ biset $V_{y x}$ to be $L_{x}$ with right multiplication by $L_{x}$ as the right action and use $c_{h_{2}}: L_{y} \rightarrow L_{x}$ to define the left action. Note that $V_{y x}$ is an isomorphism and a conjugation biset. By Lemma 4.8, we have $\operatorname{Ind}_{x} \cong \operatorname{Ind}_{y} \times_{L_{y}} V_{y x}$.

Similarly, we define a $K_{y}-K_{x}$ biset $W_{y x}$ to be $K_{x}$ with right multiplication by $K_{x}$ and left multiplication defined by $c_{h_{1}}: K_{y} \rightarrow K_{x}$. We have $\operatorname{Res}_{x} \cong W_{y x}^{-1} \times_{K_{y}} \operatorname{Res}_{y}$ and $\mathfrak{L}_{x} \cong V_{y x}^{-1} \times_{L_{y}} \mathfrak{L}_{y} \times_{K_{y}} W_{y x}$. 
Definition 6.11. We define three subcategories of B. $(G)$, denoted $\operatorname{Ind}_{\mathbf{B}}(G) \subset \operatorname{Ind}_{\mathbf{A}}(G)$, $\operatorname{Res}_{\mathbf{B}}(G) \subset \operatorname{Res}_{\mathbf{A}}(G)$ and $\operatorname{Iso}_{\mathbf{B}}(G) \subset \operatorname{Iso}_{\mathbf{A}}(G)$. These are the subcategories with the same objects as the bigger categories, but whose morphisms are all the morphisms which are conjugation bisets.

\section{Bifunctors into B. $(G)$}

In this section we will define the bivariant functor $j: \mathcal{D}(G) \rightarrow \mathbf{B}_{\bullet}(G)$ used in the statement of Theorem A. Let $\mathcal{D}_{*}(G)$ denote the category whose objects are pairs $(X, \mathbf{b})$, consisting of a finite $G$-space $X$ and an ordered collection $\mathbf{b}=\left(b_{1}, \cdots, b_{n}\right)$ of base-points, one for each $G$-orbit of $X$. The morphisms are the $G$-maps (not necessarily base-point preserving). There is a functor

$$
\mu: \mathcal{D}_{*}(G) \rightarrow \mathcal{D}(G)
$$

defined by forgetting the base-points. Since every object of $\mathcal{D}(G)$ is isomorphic to the image $\mu(X, \mathbf{b})$ of an object of $\mathcal{D}_{*}(G)$, and $\mu$ induces a bijection on morphism sets, it follows that $\mu$ gives an equivalence between the categories $\mathcal{D}_{*}(G)$ and $\mathcal{D}(G)$, with inverse functor $\mu^{\prime}$ [16, IV.4, Theorem 1, p. 91]. Moreover, the inverse functor $\mu^{\prime}$ can be chosen so that we have the additivity formula

$$
\mu^{\prime}(X \sqcup Y)=\mu^{\prime}(X) \sqcup \mu^{\prime}(Y)
$$

for any finite $G$-sets $X$ and $Y$. This will be needed later to verify axiom (M2) for additive functors out of $\mathbf{B} \cdot(G)$.

We will now define the remaining functors in the following diagram:

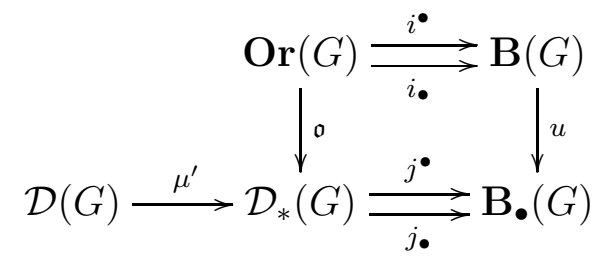

The functor $\mathfrak{o}: \operatorname{Or}(G) \rightarrow \mathcal{D}_{*}(G)$ sends $H$ to the 1 -tuple $(G / H, e H)$ and a $G$-set map $G / H \rightarrow G / K$ to the same $G$-set map. In fact $\operatorname{Or}(G)$ as defined is isomorphic to a full subcategory of $\mathcal{D}_{*}(G)$.

The functor $i$ is the identity on objects and sends a $G$-map $f: G / H \rightarrow G / K$ to the conjugation biset ${ }_{K} K_{g^{-1} H g}$ where $f(e H)=g K$. This is well-defined, since a different choice $g k$, for $k \in K$, of representative yields an isomorphic biset.

Note that $1_{G / H}: G / H \rightarrow G / H$ goes to ${ }_{H} H_{H}$ which is the identity. Check that if $f_{1}: G / H_{1} \rightarrow G / H_{2}$ and $f_{2}: G / H_{2} \rightarrow G / H_{3}$ are $G$-maps and if $f_{1}\left(e H_{1}\right)=g_{1} H_{2}$ and $f_{2}\left(e H_{2}\right)=g_{2} H_{3}$ then $f_{2} \circ f_{1}\left(e H_{1}\right)=\left(g_{1} g_{2}\right) H_{3}$ and

$$
H_{3}\left(H_{3}\right)_{g_{2}^{-1} H_{2} g_{2}} \times H_{2} H_{2}\left(H_{2}\right)_{g_{1}^{-1} H_{1} g_{1}}
$$

is isomorphic to $H_{3}\left(H_{3}\right)_{\left(g_{1} g_{2}\right)^{-1} H_{1}\left(g_{1} g_{2}\right)}$ by the map $\left(h_{3}, h_{2}\right) \mapsto h_{3} g_{2}^{-1} h_{2} g_{2}$.

The functor $i \bullet$ is also the identity on objects, but sends a $G$-map $f: G / H \rightarrow G / K$ to the conjugation biset $g^{-1}{ }_{g} K_{K}$ where $f(e H)=g K$. Rather than check identity and 
composition, just note that ${ }_{g^{-1} H g} K_{K}$ is isomorphic to $\tau\left({ }_{K} K_{g^{-1} H g}\right)$ by the function which sends $k$ to $k^{-1}$, so $i^{\bullet}=\tau \circ i_{\bullet}$ and hence $i^{\bullet}$ is a contravariant functor.

We define the functor $j_{\bullet}$ on objects by additivity: every object of $\mathcal{D}_{*}(G)$ has the form

$$
(X, \mathbf{b})=\bigsqcup_{i=1}^{k}\left(X_{i}, b_{i}\right),
$$

where $\left(X_{i}, b_{i}\right)=G / H_{i}$ is an object of $\operatorname{Or}(G)$, and we send such an object to the ordered $n$-tuple $\left(i_{\bullet}\left(H_{1}\right), \ldots, i_{\bullet}\left(H_{k}\right)\right)$. A morphism $\varphi:(X, \mathbf{b}) \rightarrow(Y, \mathbf{c})$ in $\mathcal{D}_{*}(G)$ is a collection $\left(\varphi_{i}\right), 1 \leqslant i \leqslant k$, of $G$-maps of the form $\varphi_{i}: G / H_{i} \rightarrow G / K_{f(i)}$, where $f: \mathbf{b} \rightarrow \mathbf{c}$ is a function. Every morphism in B. $(G)$ is represented a finite matrix of bifree conjugation bisets, where $\emptyset=0$. We define $j_{\bullet}(\varphi)=\alpha$ to be the morphism in $\mathbf{B}_{\bullet}(G)$ represented by the matrix $\alpha=\left(\alpha_{i j}\right)$, where $\alpha_{i j}=i_{\bullet}\left(\varphi_{i}\right)$, if $j=f(i)$, and $\alpha_{i j}=0$ otherwise.

The functor $j^{\bullet}$ is defined in a similar way. Notice that $j \bullet$ factors through the subcategory $\operatorname{Ind}_{\mathbf{B}}(G)$, and $j^{\bullet}$ factors through the subcategory $\operatorname{Res}_{\mathbf{B}}(G)$.

Definition 7.2. We define the bivariant functor

$$
j: \mathcal{D}(G) \rightarrow \mathbf{B}_{\bullet}(G)
$$

as the composition $j=\left(j^{\bullet}, j_{\bullet}\right) \circ \mu^{\prime}$.

\section{The Proof of Theorem A}

We now state our main result, which is a more detailed version of Theorem A.

Theorem 8.1. If $F: \mathbf{B}_{\bullet}(G) \rightarrow \mathcal{A} b$ is an additive functor, then $F \circ j: \mathcal{D}(G) \rightarrow \mathcal{A} b$ is a conjugation invariant Mackey functor. Conversely, any conjugation invariant Mackey functor factors uniquely through an additive functor out of $\mathbf{B} \cdot(G)$.

Proof. Suppose that $F:$ B. $(G) \rightarrow \mathcal{A} b$ is an additive functor, and let $\mathcal{M}=F \circ j: \mathcal{D}(G) \rightarrow$ $\mathcal{A} b$ denote its composition with the bivariant functor $j$. Then $\mathcal{M}$ is conjugation-invariant. The Mackey property (M2) is just additivity, so it remains to consider (M1). Let

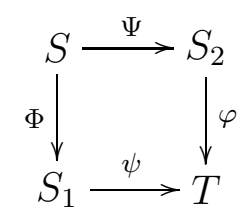

be a pull-back diagram of finite $G$-sets. We first remark that a $G$-map $u: S \rightarrow S^{\prime}$ between finite $G$-sets is determined by its restriction to the disjoint $G$-orbits in $S$. In fact, each orbit in $S$ is mapped by $u$ into exactly one $G$-orbit of $S^{\prime}$. In particular, if $u: S \rightarrow S^{\prime}$ is an isomorphism of finite $G$-sets, then by Proposition 6.4 the induced maps $u_{\mathcal{M}}$ and $u^{\mathcal{M}}$ are both isomorphisms with $\left(u_{\mathcal{M}}\right)^{-1}=u^{\mathcal{M}}$.

This remark implies that property (M1) depends only on the isomorphism class of diagram (8.2). More precisely, let $c: S_{1} \underset{\sim}{\stackrel{\sim}{\rightarrow}} S_{1}^{\prime}$ and $d: S_{2} \stackrel{\approx}{\rightarrow} S_{2}^{\prime}$ be $G$-isomorphisms, and 
consider the commutative diagram of $G$-sets and $G$-maps:

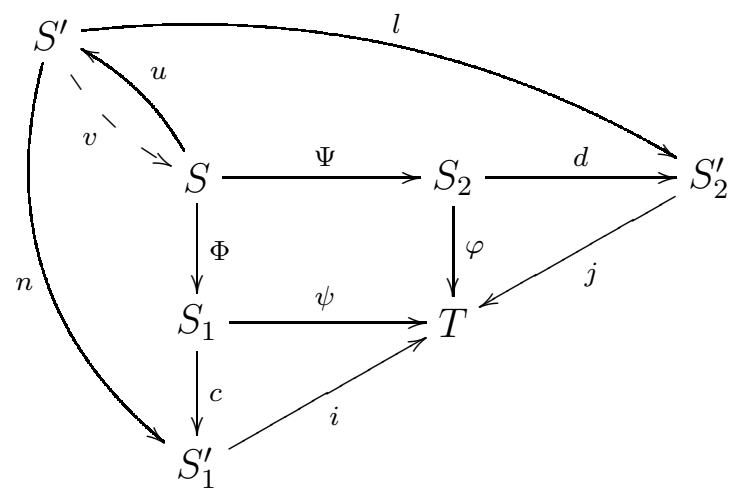

where $S^{\prime}$ is the pullback of $i$ and $j$, with $\psi=i \circ c$ and $\varphi=j \circ d$. There is an induced isomorphism $u: S \rightarrow S^{\prime}$ with inverse $v$, such that $l \circ u=d \circ \Psi$, and $c \circ \Phi=n \circ u$. If we have the property (M1) for the pullback $\left(S^{\prime}, l, n\right)$, then the property (M1) holds for the pullback $(S, \Psi, \Phi)$. This is an easy diagram chase starting with the left-hand side of the required formula

$$
\varphi^{\mathcal{M}} \circ \psi_{\mathcal{M}}=\Psi_{\mathcal{M}} \circ \Phi^{\mathcal{M}}
$$

and substituting in the expressions above for $\psi$ and $\varphi$.

By the additivity property (M2), this formula follows from the basic cases where $S_{1}$, $S_{2}$, and $T$ are transitive $G$-sets. Let $S_{1}=G / H_{1}, S_{2}=G / H_{2}$ and $T=G / K$, and choose elements $g_{1}, g_{2} \in G$ such that $g_{i}^{-1} H_{i} g_{i} \subseteq K$, for $i=1,2$. Since $\mathcal{M}$ is conjugation invariant, we may assume that $\psi\left(e H_{1}\right)=g_{1} K$ and $\varphi\left(e H_{2}\right)=g_{2} K$ (varying the choice of $g_{1}, g_{2}$ will not affect the maps induced by $\mathcal{M})$.

For $i=1$ and $i=2$ there are $G$-isomorphisms $\theta_{i}: G / H_{i} \rightarrow G / H_{i}^{\prime}$, given by $e H_{i} \mapsto g_{i} H_{i}^{\prime}$ where $H_{i}^{\prime}=g_{i}^{-1} H_{i} g_{i}$, with the property that the pullback $G / H_{1}^{\prime} \subset G / K \supset G / H_{2}^{\prime}$ is isomorphic to $S=G / H_{1} \times_{G / K} G / H_{2}$. It follows (by the remarks above) that it is enough to check formula (8.3) in the special case where $H_{1}$ and $H_{2}$ are actually subgroups of $K$. Hence, we may assume that $g_{1}=g_{2}=e$.

We will regard ${ }_{H_{2}} K_{H_{1}}$ as an $H_{2}-H_{1}$ biset via the natural actions $H_{2} \subset K$ and $H_{1} \subset K$. The pullback $G$-set

$$
S=\left\{\left(x H_{1}, y H_{2}\right) \mid x K=y K\right\}
$$

has the $G$-action defined by $g \cdot\left(x H_{1}, y H_{2}\right)=\left(g x H_{1}, g y H_{2}\right)$, for all $g \in G$. It follows that in each $G$-orbit there is always a representative of the form $\left(x H_{1}, e H_{2}\right)$ with $x \in K$ and $\left(h_{2} x h_{1} H_{1}, e H_{2}\right)=\left(x H_{1}, e H_{2}\right)$ for all $h_{2} \in H_{2}$ and all $h_{1} \in H_{1}$. In other words, the set of $G$-orbits in $S$ is in bijection with the quotient $H_{2} \backslash K / H_{1}$ of the biset ${ }_{H_{2}} K_{H_{1}}$.

The isotropy subgroup

$$
G_{\left(x H_{1}, e H_{2}\right)}=\left\{g \in G \mid g x H_{1}=x H_{1}, g H_{2}=e H_{2}\right\}=H_{2} \cap H_{1}^{x^{-1}}
$$

where $H_{1}^{x^{-1}}=x H_{1} x^{-1}$ and $x \in K$ as above. Therefore, we have a bijection of $G$-sets

$$
S=\bigsqcup_{x \in H_{2} \backslash K / H_{1}} G / H_{2} \cap H_{1}^{x^{-1}} .
$$


In terms of biset morphisms, the composition

$$
\Psi_{\mathcal{M}} \circ \Phi^{\mathcal{M}}=\sum F\left({ }_{H_{2}} H_{2}{ }_{H_{2} \cap H_{1}^{x}}{ }^{-1}\right) \circ F\left({ }_{H_{2} \cap H_{1}^{x}}{ }^{-1}\left(H_{2} \cap H_{1}^{x^{-1}}\right)_{H_{1} \cap H_{2}^{x}}\right) \circ F\left({ }_{H_{1} \cap H_{2}^{x}} H_{1_{H_{1}}}\right)
$$

where the middle biset is just the conjugation $G$-isomorphism $c_{x^{-1}}: H_{1} \cap H_{2}^{x} \rightarrow H_{2} \cap H_{1}^{x^{-1}}$.

On the other hand, the composition

$$
\varphi^{\mathcal{M}} \circ \psi_{\mathcal{M}}=F\left({ }_{H_{2}} K_{H_{1}}\right) .
$$

To establish formula (8.3), we consider the $H_{2}-H_{1}$ biset bijection

$$
{ }_{H_{2}} K_{H_{1}}=\bigsqcup_{x \in H_{2} \backslash K / H_{1}} H_{2} x H_{1}
$$

from the double coset decompostion. The standard representation of the component $X=H_{2} x H_{1}$ expressed in the standard form is

$$
H_{2} x H_{1}={ }_{H_{2}}\left[L_{x}, \gamma_{X, x}, K_{x}\right]_{H_{1}}=\left[H_{2} \cap H_{1}^{x^{-1}}, c_{x}, H_{1} \cap H_{2}^{x}\right] .
$$

This follows from Remark 4.6: $L_{x}=\left\{h_{2} \in H_{2} \mid h_{2} x=x h_{1}, h_{1} \in H_{1}\right\}=H_{2} \cap H_{1}^{x^{-1}}$, $K_{x}=\left\{h_{1} \in H_{1} \mid x h_{1}=h_{2} x, h_{2} \in H_{2}\right\}=H_{1} \cap H_{2}^{x}$ and $\gamma_{X, x}=c_{x}$.

Now by Proposition 6.9 we see that the composition

$$
\varphi^{\mathcal{M}} \circ \psi_{\mathcal{M}}=F\left({ }_{H_{2}} K_{H_{1}}\right)=\sum F\left(\operatorname{Ind}_{x}\right) \circ F\left(\mathfrak{L}_{x}\right) \circ F\left(\operatorname{Res}_{x}\right) .
$$

But, by inspection, the right-hand side of this formula is just the expression for $\Psi_{\mathcal{M}} \circ \Phi^{\mathcal{M}}$ in formula (8.4). This proves the property (M1).

Conversely, suppose that $\mathcal{M}: \mathcal{D}(G) \rightarrow \mathcal{A} b$ is a conjugation-invariant Mackey functor. We define the functor $F$ on objects by setting $F(H)=\mathcal{M}(G / H)$, and extending additively. Since the bivariant functor $j: \mathcal{D}(G) \rightarrow \mathbf{B} \bullet(G)$ is surjective on objects, this formula defines $F$ uniquely on objects.

The morphisms in B. $(G)$ are finite matrices of bifree conjugation $H_{2}-H_{1}$ bisets. Any such biset is a disjoint union of indecomposable conjugation bisets $X$, uniquely up to ordering, and after picking a base point $x \in X$ we have the standard representation $X \cong{ }_{H_{2}}\left[L_{x}, \gamma_{X, x}, K_{x}\right]_{H_{1}}$ and the factorization

$$
{ }_{H_{2}} X_{H_{1}} \cong{ }_{H_{2}}\left[L_{x}, \gamma_{X, x}, K_{x}\right]_{H_{1}} \cong \operatorname{Ind}_{x} \circ \mathfrak{L}_{x} \circ \operatorname{Res}_{x}
$$

of Proposition 6.9. By Definition 6.8, it follows that the morphisms in B. $(G)$ are generated by compositions of the following three types of bifree conjugation bisets:

$$
\text { (i) } \operatorname{Ind}_{x}={ }_{H_{2}}\left(H_{2}\right)_{L_{x}}, \quad \text { (ii) } \operatorname{Res}_{x}={ }_{K_{x}}\left(H_{1}\right)_{H_{1}}, \quad \text { and } \quad \text { (iii) } \mathfrak{L}_{x}={ }_{L_{x}}\left(L_{x}\right)_{K_{x}} \text {. }
$$

Recall from Remark 4.6 that

$$
L_{x}=\left\{h_{2} \in H_{2} \mid h_{2} x=x h_{1}, h_{1} \in H_{1}\right\} \subset H_{2}
$$

and

$$
K_{x}=\left\{h_{1} \in H_{1} \mid x h_{1}=h_{2} x, h_{2} \in H_{2}\right\} \subset H_{1} .
$$

Since $X$ is a conjugation biset, the map $\gamma_{X, x}: L_{x} \rightarrow K_{x}$ is given by $c_{g_{x}}$, where $g_{x}^{-1} L_{x} g_{x}=$ $K_{x}$, so we have

$$
\gamma_{X, x}\left(h_{2}\right)=c_{g_{x}}\left(h_{2}\right)=g_{x}^{-1} h_{2} g_{x}=h_{1}
$$


The element $g_{x}$ need not be unique (see Remark 6.2). The isomorphism $\gamma_{X, x}^{-1}$ gives $L_{x}$ the right $K_{x}$-action on $L_{x}$ used to define the third biset.

We will define $F$ on the basic morphisms of these three types, by associating to each of these bisets a $G$-map, and then applying the Mackey functor $\mathcal{M}$ :

- to $\operatorname{Ind}_{x}$ associate the $G$-map $\operatorname{Ind}_{L_{x}}^{H_{2}}: G / L_{x} \rightarrow G / H_{2}$, with $\operatorname{Ind}_{L_{x}}^{H_{2}}\left(e L_{x}\right)=e H_{2}$;

- to $\operatorname{Res}_{x}$ associate the $G$-map $\operatorname{Res}_{K_{x}}^{H_{1}}: G / K_{x} \rightarrow G / H_{1}$, with $\operatorname{Res}_{K_{x}}^{H_{1}}\left(e K_{x}\right)=e H_{1}$;

- to $\mathfrak{L}_{x}$ associate the maps of $G$-sets $\mathfrak{L}_{K_{x}}^{L_{x}}\left(g_{x}\right): G / K_{x} \rightarrow G / L_{x}$, with $\mathfrak{L}_{K_{x}}^{L_{x}}\left(g_{x}\right)\left(e K_{x}\right)=$ $g_{x}^{-1} L_{x}$ for the various choices of $g_{x}$.

Now define

(i) $F\left(\operatorname{Ind}_{x}\right)=\mathcal{M}_{*}\left(\operatorname{Ind}_{L_{x}}^{H_{2}}\right)$

(ii) $F\left(\operatorname{Res}_{x}\right)=\mathcal{M}^{*}\left(\operatorname{Res}_{K_{x}}^{H_{1}}\right)$

(iii) $F\left(\mathfrak{L}_{x}\right)=\mathcal{M}_{*}\left(\mathfrak{L}_{K_{x}}^{L_{x}}\left(g_{x}\right)\right)$

Item (iii) is well-defined by Lemma 3.2 , since $\mathcal{M}$ is conjugation invariant. We will write $\mathfrak{L}_{K_{x}}^{L_{x}}$ for any of the $\mathfrak{L}_{K_{x}}^{L_{x}}\left(g_{x}\right)$.

For any morphism $X$ in $\mathbf{B}(G)$ we define $F(X)$ by choosing a base point $x \in X$, writing $X=\operatorname{Ind}_{x} \circ \mathfrak{L}_{x} \circ \operatorname{Res}_{x}$, and defining $F(X)=F\left(\operatorname{Ind}_{x}\right) \circ F\left(\mathfrak{L}_{x}\right) \circ F\left(\operatorname{Res}_{x}\right)$. The formulas in Remark 6.10 show that the definition of $F(X)$ is independent of the choice of base point.

The functor $F$ is defined on all the morphisms in B. $(G)$ by the additive (matrix) extension of these formulas. Any relation in the Grothendieck group $\operatorname{Hom}_{\mathbf{B}(G)}\left(H_{1}, H_{2}\right)$ leads to a isomorphism $X \cong Y$ of finite bifree $H_{2}-H_{1}$ bisets. Since both sides are canonically (up to ordering) expressed as a disjoint union of $\left(H_{2} \times H_{1}^{o p}\right)$-orbits, it is clear that $F$ is well-defined.

Finally, we must check that $F$ is a functor. Since $F\left({ }_{H} H_{H}\right)=i d$, it remains to check that compositions are preserved. Suppose that $X_{1} \in \operatorname{Hom}_{\mathbf{B}(G)}\left(H_{1}, H_{2}\right)$ and $X_{2} \in$ $\operatorname{Hom}_{\mathbf{B}(G)}\left(H_{2}, H_{3}\right)$. By additivity, we may assume that $X_{1}$ and $X_{2}$ are indecomposable. We must check that

$$
F\left(X_{2} \circ X_{1}\right)=F\left(X_{2}\right) \circ F\left(X_{1}\right) .
$$

Consider the left-hand side of the formula, where $F\left(X_{2} \circ X_{1}\right)=F\left(X_{2} \times_{H_{2}} X_{1}\right)$ by definition. Pick a base point $x_{1} \in X_{1}$, and $x_{2} \in X_{2}$. By Lemma 5.2, the components of $X_{3}=$ $X_{2} \times_{H_{2}} X_{1}$ are indexed by elements $h_{2} \in H_{2}$ representing the double cosets $K_{x_{2}} \backslash H_{2} / L_{x_{1}}$. Each such component contributes a summand $F\left(\left[L_{x_{3}}, \gamma_{x_{3}, x_{3}}, K_{x_{3}}\right]\right)$, where $x_{3}=\left[x_{2} h_{2}, x_{1}\right]$. By Theorem 5.3, the standard representation at this base point is

(8.5) $. ~\left[L_{x_{3}}, \gamma_{X_{3}, x_{3}}, K_{x_{3}}\right]=\left[\gamma_{X_{2}, x_{2}}^{-1}\left(K_{x_{2}} \cap L_{x_{1}}^{h_{2}^{-1}}\right), \gamma_{X_{1}, x_{1}} \circ c_{h_{2}} \circ \gamma_{X_{2}, x_{2}}, \gamma_{X_{1}, x_{1}}\left(K_{x_{2}}^{h_{2}} \cap L_{x_{1}}\right)\right]$

The right-hand side of the formula is

$$
F\left(X_{2}\right) \circ F\left(X_{1}\right)=F\left(\operatorname{Ind}_{x_{2}}\right) \circ F\left(\mathfrak{L}_{x_{2}}\right) \circ F\left(\operatorname{Res}_{x_{2}}\right) \circ F\left(\operatorname{Ind}_{x_{1}}\right) \circ F\left(\mathfrak{L}_{x_{1}}\right) \circ F\left(\operatorname{Res}_{x_{1}}\right) .
$$

By the Mackey double coset formula for $\mathcal{M}$, property (M1),

$$
F\left(\operatorname{Res}_{x_{2}}\right) \circ F\left(\operatorname{Ind}_{x_{1}}\right)=\sum_{h_{2} \in K_{x_{2}} \backslash H_{2} / L_{x_{1}}} F\left(K_{x_{2}}\right) \circ F\left(K_{x_{2}} \cap L_{x_{1}}^{h_{2}^{-1}}\right) \circ F\left(L_{x_{1}}\right)
$$

where

- $K_{x_{2}}$ is a $\left(K_{x_{2}}\right)-\left(K_{x_{2}} \cap L_{x_{1}}^{h_{2}^{-1}}\right)$ biset via the two inclusions, and is a standard induction, 
- $K_{x_{2}} \cap L_{x_{1}}^{h_{2}^{-1}}$ is a $\left(K_{x_{2}} \cap L_{x_{1}}^{h_{2}^{-1}}\right)-\left(L_{x_{1}} \cap K_{x_{2}}^{h_{2}}\right)$ biset via the evident inclusion on the left and conjugation by $h_{2}^{-1}$ on the right, and is a standard conjugation,

- $L_{x_{1}}$ is an $\left(L_{x_{1}} \cap K_{x_{2}}^{h_{2}}\right)$ - $\left(L_{x_{1}}\right)$ biset via the two inclusions and is a standard restriction.

Hence $F\left(X_{2}\right) \circ F\left(X_{1}\right)=\sum_{h_{2} \in K_{x_{2}} \backslash H_{2} / L_{x_{1}}} A\left[h_{2}\right] \circ B\left[h_{2}\right] \circ C\left[h_{2}\right]$. where

$$
\begin{aligned}
& A\left[h_{2}\right]=\mathcal{M}_{*}\left(\operatorname{Ind}_{L_{x_{2}}}^{H_{3}}\right) \circ \mathcal{M}_{*}\left(\mathfrak{L}_{K_{x_{2}}}^{L_{x_{2}}}\right) \circ \mathcal{M}_{*}\left(\begin{array}{c}
\operatorname{Ind}_{K_{x_{2}} \cap L_{x_{1}}^{h_{2}}}^{K_{x_{2}}}
\end{array}\right) \\
& B\left[h_{2}\right]=\mathcal{M}_{*}\left(\mathfrak{L}_{L_{x_{1}} \cap K_{x_{2}}^{h_{2}}}^{K_{x_{2}} \cap L_{2}^{h_{2}^{-1}}}\right) \\
& C\left[h_{2}\right]=\mathcal{M}^{*}\left(\operatorname{Res}_{L_{x_{1}} \cap K_{x_{2}}^{h_{2}}}^{L_{x_{1}}}\right) \circ \mathcal{M}_{*}\left(\mathfrak{L}_{K_{x_{1}}}^{L_{x_{1}}}\right) \circ \mathcal{M}^{*}\left(\operatorname{Res}_{K_{x_{1}}}^{H_{1}}\right)
\end{aligned}
$$

To further analyze $A\left[h_{2}\right]$ consider the commutative diagram

$$
\begin{array}{ccc}
H_{3} \supset L_{x_{2}} & \stackrel{\gamma_{X_{2}, x_{2}}^{-1}}{ } & K_{x_{2}} \\
\cup & & \cup \\
\gamma_{X_{2}, x_{2}}^{-1}\left(K_{x_{2}} \cap L_{x_{1}}^{h_{2}^{-1}}\right) & \stackrel{\gamma_{X_{2}, x_{2}}^{-1}}{\longleftarrow} & K_{x_{2}} \cap L_{x_{1}}^{h_{2}^{-1}}
\end{array}
$$

which implies

$$
A\left[h_{2}\right]=\mathcal{M}_{*}\left(\begin{array}{c}
\operatorname{Ind}^{H_{3}} \\
\gamma_{X_{2}, x_{2}}^{-1}\left(K_{x_{2}} \cap L_{x_{1}}^{h_{2}^{-1}}\right)
\end{array}\right) \circ \mathcal{M}_{*}\left(\begin{array}{c}
\left.\mathfrak{L}_{\mathcal{X}_{2}, x_{2}}^{\gamma_{x_{2}}^{-1}\left(K_{x_{2}} \cap L_{x_{1}}^{h_{2}^{-1}}\right)}\right) \\
K_{x_{1}}^{h_{2}^{-1}}
\end{array}\right)
$$

To analyze $C\left[h_{2}\right]$ first note that $\mathcal{M}_{*}\left(\mathfrak{L}_{K_{x_{1}}}^{L_{x_{1}}}\right)=\mathcal{M}^{*}\left(\mathfrak{L}_{L_{x_{1}}}^{K_{x_{1}}}\right)$. Then consider the commutative diagram

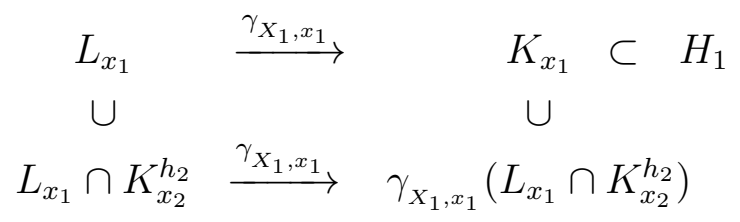

which implies

$$
\begin{aligned}
& C\left[h_{2}\right]=\mathcal{M}^{*}\left(\operatorname{Res}_{L_{x_{1}} \cap K_{x_{2}}^{h_{2}}}^{L_{x_{1}}}\right) \circ \mathcal{M}^{*}\left(\mathfrak{L}_{L_{x_{1}}}^{K_{x_{1}}}\right) \circ \mathcal{M}^{*}\left(\operatorname{Res}_{K_{x_{1}}}^{H_{1}}\right) \\
& =\mathcal{M}^{*}\left(\mathfrak{L}_{L_{x_{1}} \cap K_{x_{2}}^{h_{2}}}^{\gamma_{X_{1}, x_{1}}\left(L_{x_{1}} \cap K_{x_{2}}^{h_{2}}\right)}\right) \circ \mathcal{M}^{*}\left(\operatorname{Res}_{\gamma_{X_{1}, x_{1}} H_{1}}^{\left.H_{x_{1}} \cap K_{x_{2}}^{h_{2}}\right)}\right)
\end{aligned}
$$

To analyze the conjugations, note that after substituting the new expressions for $A\left[h_{2}\right]$ 
and $C\left[h_{2}\right]$ we have three conjugations now occurring together in $A\left[h_{2}\right] \circ B\left[h_{2}\right] \circ C\left[h_{2}\right]$ :

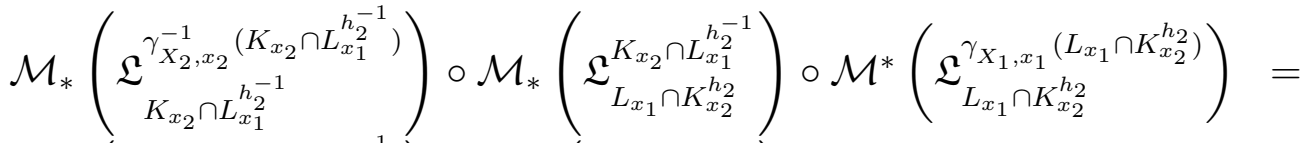

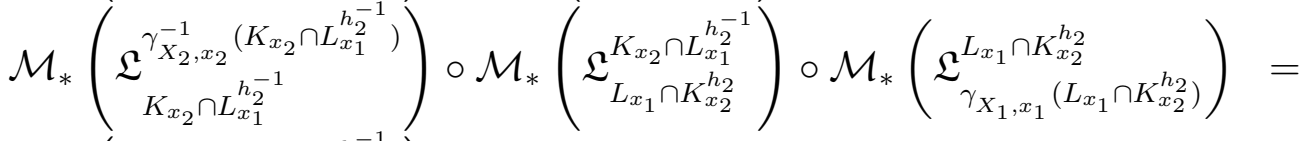

$$
\begin{aligned}
& \mathcal{M}_{*}\left(\mathfrak{L}_{\gamma_{X_{1}, x_{1}}^{\gamma_{X_{2}}}\left(L_{x_{1}} \cap K_{x_{2}}^{h_{2}}\right)}^{h_{x_{2}}}\right)
\end{aligned}
$$

Hence

$$
\begin{aligned}
& A\left[h_{2}\right] \circ B\left[h_{2}\right] \circ C\left[h_{2}\right]=
\end{aligned}
$$

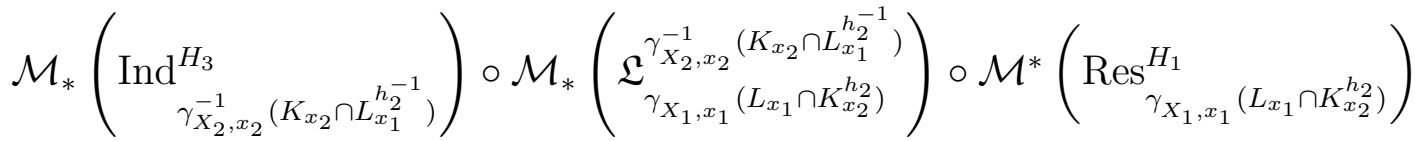

It now follows from $(8.5)$ that

$$
A\left[h_{2}\right] \circ B\left[h_{2}\right] \circ C\left[h_{2}\right]=F\left(\operatorname{Ind}_{x_{3}}\right) \circ F\left(\mathfrak{L}_{x_{3}}\right) \circ F\left(\operatorname{Res}_{x_{3}}\right)
$$

for each component $x_{3}=\left[x_{2} h_{2}, x_{1}\right]$. Therefore $F\left(X_{2} \circ X_{1}\right)=F\left(X_{2}\right) \circ F\left(X_{1}\right)$.

\section{REFERENCES}

[1] J. F. Adams, J. H. Gunawardena, and H. Miller, The Segal conjecture for elementary abelian p-groups, Topology 24 (1985), 435-460.

[2] S. Bouc, Foncteurs d'ensembles munis d'une double action, J. Algebra 183 (1996), no. 3, 664-736 (French).

[3] _ Biset functors and genetic sections for p-groups, J. Algebra 284 (2005), 179-202.

[4] _ Rational p-biset functors, J. Algebra 319 (2008), 1776-1800.

[5] S. Bouc and J. Thévenaz, The group of endo-permutation modules, Invent. Math. 139 (2000), no. $2,275-349$.

[6] _ A sectional characterization of the Dade group, J. Group Theory 11 (2008), no. 2, 155183.

[7] T. tom Dieck, Transformation groups, de Gruyter Studies in Mathematics, vol. 8, Walter de Gruyter \& Co., Berlin, 1987.

[8] A. W. M. Dress, Induction and structure theorems for orthogonal representations of finite groups, Ann. of Math. 102 (1975), 291-325.

[9] J. A. Green, Axiomatic representation theory for finite groups, J. Pure Appl. Algebra 1 (1971), no. $1,41-77$.

[10] I. Hambleton, L. R. Taylor, and E. B. Williams, Detection theorems for K-theory and L-theory, J. Pure Appl. Algebra 63 (1990), 247-299.

[11] _ Induction theory, 1990, MSRI Preprint 05425-90.

[12] Dress Induction and the Burnside quotient Green ring, Algebra \& Number Theory 3 (2009), 511-541.

[13] L. G. Lewis, The theory of Green functors, 1980, mimeographed notes.

[14] L. G. Lewis, J. P. May, and J. E. McClure, Classifying G-spaces and the Segal conjecture, Current trends in algebraic topology, Part 2 (London, Ont., 1981), CMS Conf. Proc., vol. 2, Amer. Math. Soc., Providence, R.I., 1982, pp. 165-179.

[15] H. Lindner, A remark on Mackey-functors, Manuscripta Math. 18 (1976), 273-278. 
[16] S. Mac Lane, Categories for the working mathematician, 2nd ed., Graduate Texts in Mathematics, vol. 5, Springer-Verlag, New York, 1998.

[17] J. Thévenaz and P. Webb, The structure of Mackey functors, Trans. Amer. Math. Soc. 347 (1995), 1865-1961.

[18] P. Webb, Two classifications of simple Mackey functors with applications to group cohomology and the decomposition of classifying spaces, J. Pure Appl. Algebra 88 (1993), no. 1-3, 265-304.

[19] - A guide to Mackey functors, Handbook of algebra, Vol. 2, North-Holland, Amsterdam, 2000 , pp. 805-836.

Department of Mathematics \& Statistics

McMaster University

Hamilton, ON L8S 4K1, Canada

E-mail address: ian@math.mcmaster.ca

Department of Mathematics

University of Notre Dame

Notre Dame, IN 46556, USA

E-mail address: taylor.2@nd.edu

Department of Mathematics

University of Notre Dame

Notre Dame, IN 46556, USA

E-mail address: williams.4@nd.edu 